\title{
Capacity Bounds for Discrete-Time, Amplitude-Constrained, Additive White Gaussian Noise Channels
}

\author{
Andrew Thangaraj, Gerhard Kramer and Georg Böcherer
}

\begin{abstract}
The capacity-achieving input distribution of the discrete-time, additive white Gaussian noise (AWGN) channel with an amplitude constraint is discrete and seems difficult to characterize explicitly. A dual capacity expression is used to derive analytic capacity upper bounds for scalar and vector AWGN channels. The scalar bound improves on McKellips' bound and is within 0.1 bits of capacity for all signal-to-noise ratios (SNRs). The two-dimensional bound is within 0.15 bits of capacity provably up to $4.5 \mathrm{~dB}$, and numerical evidence suggests a similar gap for all SNRs.
\end{abstract}

Index Terms-additive white Gaussian noise channel, amplitude constraint, capacity

\section{INTRODUCTION}

The most commonly-studied channel model for communications is the additive white Gaussian noise (AWGN) channel. The AWGN model is interesting only with constraints on the channel input or output. Depending on the application, one is interested in limiting, e.g., the average input (or output) variance or the input amplitude.

Input (or output) variance constraints result in elegant analytic capacity expressions such as Shannon's $\frac{1}{2} \log (1+\mathrm{SNR})$ formula. The amplitude constraint seems less tractable, and typical analyses use Smith's methods [1] to show that the capacity-achieving input distribution has discrete amplitudes, see [2], [3], [4], [5] and references therein. A recent line of work studies the peak-to-average power (PAPR) ratio of good codes [6].

An alternative approach is by McKellips [7] who develops analytic and tight capacity upper bounds by bounding the channel output entropy. We instead use the dual capacity expression in [8, p. 128] (see also [9, Eqn. (7)]) and study both scalar and vector channels. The dual approach was also used in [10] for scalar AWGN channels with non-negative channel inputs. Our models and results differ from those in [10]: we do not impose a non-negativity constraint (this difference turns out to be minor for the scalar case), we study vector channels that include the important practical case of two-dimensional (complex) AWGN channels, and we develop certain bounds

A. Thangaraj is with the Department of Electrical Engineering, Indian Institute of Technology Madras, Chennai 600036, India, Email: andrew@ee.iitm.ac.in

G. Kramer and G. Böcherer are with the Department of Electrical and Computer Engineering, Technical University of Munich, $\mathrm{Ar}$ cisstraße 21, 80333 München, Germany, Email: gerhard.kramer@tum.de, georg.boecherer@tum.de

This paper was presented in part at the IEEE International Symposium on Information Theory (ISIT) 2015, Hong Kong. in more detail. Our bounds are within 0.15 bits of capacity provably up to $4.5 \mathrm{~dB}$, and numerically for all SNRs for twodimensional (complex) AWGN channels.

This paper is organized as follows. Section $\Pi$ presents functions, integrals, and bounds that we need later. Sections IIIV develop the one-, two-, and $n$-dimensional bounds, as well as two refinements. The appendices contain technical proofs.

\section{PRELIMINARIES}

Consider the following functions:

$$
\begin{aligned}
& \psi(x) \stackrel{(a)}{=} \frac{1}{\sqrt{2 \pi}} e^{-x^{2} / 2} \\
& Q(x) \stackrel{(b)}{=} \int_{x}^{\infty} \psi(z) d z \\
& I_{0}(x) \stackrel{(c)}{=} \frac{1}{\pi} \int_{0}^{\pi} e^{x \cos \phi} d \phi \\
& Q_{1}(a, b) \stackrel{(d)}{=} \int_{b}^{\infty} z e^{-\left(z^{2}+a^{2}\right) / 2} I_{0}(a z) d z \\
& D(p \| q) \stackrel{(e)}{=} \int_{\infty}^{\infty} p(z) \log \frac{p(z)}{q(z)} d z
\end{aligned}
$$

where $(a)$ is the Gaussian density, $(b)$ is the Q-function, $(c)$ is the modified Bessel function of the first kind of integer order $0,(d)$ is the Marcum Q-function, and $(e)$ is the informational divergence between the densities $p$ and $q$. Logarithms to the base $e$ and base 2 are denoted as $\log$ and $\log _{2}$, respectively. A few useful properties are $Q_{1}(a, 0)=1$ and the bounds

$$
\frac{x}{1+x^{2}} \psi(x)<Q(x)<\frac{1}{x} \psi(x)
$$

for $x>0$ (and for $x=0$ ). We also consider the integrals:

$$
\begin{aligned}
& \int_{x}^{\infty} y \psi(y) d y=-\left.\psi(y)\right|_{x} ^{\infty}=\psi(x) \\
& \int_{x}^{\infty} y^{2} \psi(y) d y=\int_{x}^{\infty} y(-d \psi(y))=x \psi(x)+Q(x) \\
& \int_{x}^{\infty} y^{3} \psi(y) d y=\int_{x}^{\infty} y^{2}(-d \psi(y))=\left(x^{2}+2\right) \psi(x) .
\end{aligned}
$$

For sequences $f(n)$ and $g(n)$, the standard big- $O$ notation $f(n)=O(g(n))$ denotes that $|f(n)| \leq c|g(n)|$ for a constant $c$ and sufficiently large $n$ [11]. Finally, a useful upper bound on the capacity $C$ of a memoryless channel $p_{Y \mid X}(\cdot)$ is based on the dual capacity expression [8, p. 128], [9, Eqn. (7)]. The bound is

$$
C \leq \max _{x \in \mathcal{S}} D\left(p_{Y \mid X}(\cdot \mid x) \| q_{Y}(\cdot)\right)
$$




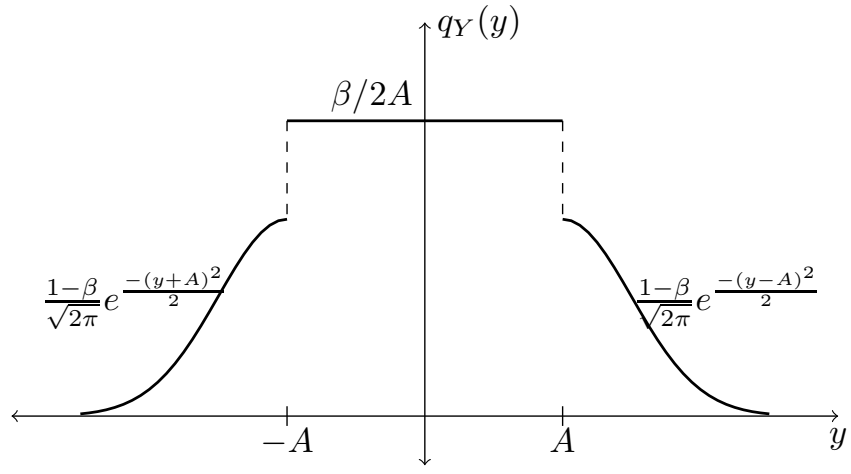

Fig. 1. Test densities for the real AWGN channel.

where $q_{Y}(\cdot)$ is any choice of "test" density $q_{Y}(\cdot)$ and $\mathcal{S}$ is the set of permitted $x$.

\section{REAL AWGN CHANNEL}

Consider the real-alphabet AWGN channel

$$
Y=X+Z
$$

where $Z$ is a Gaussian random variable with mean 0 and variance 1 . The channel density is

$$
p_{Y \mid X}(y \mid x)=\psi(y-x) .
$$

Consider the amplitude constraint $|X| \leq A$ where $A>0$. We choose a family of test densities

$$
q_{Y}(y)= \begin{cases}\frac{\beta}{2 A}, & |y| \leq A \\ \frac{1-\beta}{\sqrt{2 \pi}} e^{-(y-A)^{2} / 2}, & |y|>A\end{cases}
$$

where $\beta \in[0,1]$ is a parameter to be optimized. The test density is illustrated in Fig. 1. It is a mixture of two distributions, a uniform distribution in the interval $|y| \leq A$ and a "split and scaled" Gaussian density for $|y|>A$. The parameter $\beta$ specifies the mixing proportion.

Inserting (8) into the divergence in (5), we have

$$
\begin{aligned}
D & \left(p_{Y \mid X}(\cdot \mid x) \| q_{Y}(\cdot)\right) \\
= & \int_{-\infty}^{\infty} p_{Y \mid X}(y \mid x) \log \frac{p_{Y \mid X}(y \mid x)}{q_{Y}(y)} d y \\
= & -\log (\sqrt{2 \pi e})-\log \left(\frac{\beta}{2 A}\right)[1-Q(A+x)-Q(A-x)] \\
& -\log \left(\frac{1-\beta}{\sqrt{2 \pi}}\right)[Q(A+x)+Q(A-x)] \\
& +\frac{1}{2}\left\{\left[(A+x)^{2}+1\right] Q(A+x)-(A+x) \psi(A+x)\right. \\
& \left.+\left[(A-x)^{2}+1\right] Q(A-x)-(A-x) \psi(A-x)\right\} \\
= & \log \frac{2 A}{\beta \sqrt{2 \pi e}}+\log \frac{\beta \sqrt{2 \pi e}}{(1-\beta) 2 A}[Q(A-x)+Q(A+x)] \\
& +\frac{1}{2}[g(A-x)+g(A+x)]
\end{aligned}
$$

where $g(u) \triangleq u^{2} Q(u)-u \psi(u)$.

\section{A. McKellips' bound}

Using (1), we readily see that $g(u) \leq 0$ for $u>0$. By symmetry, we may restrict attention to $0 \leq x \leq A$ so that $g(A-x)+g(A+x) \leq 0$. Using (9), we thus have

$$
\begin{aligned}
D\left(p_{Y \mid X}(\cdot \mid x) \| q_{Y}(\cdot)\right) \leq \log \frac{2 A}{\beta \sqrt{2 \pi e}} \\
\quad+\log \frac{\beta \sqrt{2 \pi e}}{(1-\beta) 2 A}[Q(A-x)+Q(A+x)] .
\end{aligned}
$$

To recover McKellips' bound [7], we choose

$$
\beta=\frac{2 A}{\sqrt{2 \pi e}+2 A}
$$

to make the second term in (10) equal to zero, and obtain

$$
C \leq \log \left(1+\frac{2 A}{\sqrt{2 \pi e}}\right) .
$$

We now combine (12) with the capacity under the (weaker) average power constraint $\mathrm{E}\left[X^{2}\right] \leq A^{2}$. The noise power is 1 so the signal-to-noise ratio (SNR) is $P=\sqrt{A}$. We thus arrive at McKellips' bound in [7]:

$$
C \leq \min \left\{\log \left(1+\sqrt{\frac{2 P}{\pi e}}\right), \frac{1}{2} \log (1+P)\right\} .
$$

Observe that the high-SNR power loss is $10 \log _{10}(\pi e / 2) \approx$ $6.30 \mathrm{~dB}$. However, this comparison is based on equating the maximum power $P$ with the average power. Instead, if we use the uniform distribution for $X$ then the average power is $P / 3$ and the high-SNR power loss reduces to the high-SNR shaping loss of $10 \log _{10}(\pi e / 6) \approx 1.53 \mathrm{~dB}$ (see [12]).

\section{B. Refined Bound}

McKellips' bound seems tight for high SNR, roughly above $6 \mathrm{~dB}$. For low SNR, below $0 \mathrm{~dB}$, the average-constraint bound $\frac{1}{2} \log (1+P)$ is tight. For the intermediate range between 0 to $6 \mathrm{~dB}$, we derive a better bound next.

Consider $\beta$ for which $\log \frac{\beta \sqrt{2 \pi e}}{(1-\beta) 2 A}$ is positive, i.e., consider the range

$$
\beta \geq \frac{2 A}{\sqrt{2 \pi e}+2 A} .
$$

Observe that $Q(A-x)+Q(A+x)$ increases with $x$ if $x \in$ $[0, A]$, since the derivative evaluates to $\psi(A-x)-\psi(A+x)$ which is positive for $x \in[0, A]$. Thus, the RHS of (10) is maximized by setting $x=A$, and we obtain the bound

$$
\begin{aligned}
& D\left(p_{Y \mid X}(\cdot \mid x) \| q_{Y}(\cdot)\right) \leq \log \frac{2 A}{\beta \sqrt{2 \pi e}} \\
&+\log \frac{\beta \sqrt{2 \pi e}}{(1-\beta) 2 A}[1 / 2+Q(2 A)] \\
&=(1 / 2-Q(2 A)) \log \frac{2 A}{\sqrt{2 \pi e}}-(1 / 2-Q(2 A)) \log \beta \\
&-(1 / 2+Q(2 A)) \log (1-\beta) .
\end{aligned}
$$

Setting $\beta=1 / 2-Q(2 A)$, minimizes the RHS of (15). However, from (14) this choice of $\beta$ is valid only if

$$
1 / 2-Q(2 A) \geq \frac{2 A}{\sqrt{2 \pi e}+2 A}
$$




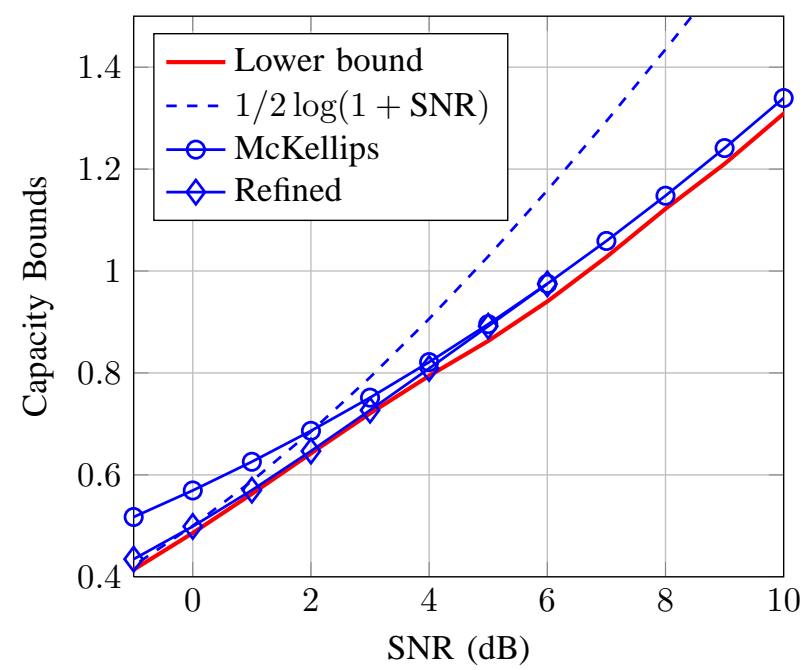

Fig. 2. Capacity bounds for scalar AWGN channels. The rate units are bits per channel use.

which is equivalent to $A \leq 2.0662 \approx \sqrt{\pi e / 2}$. Therefore, using $P=\sqrt{A}$, we have the bound

$$
C \leq \beta(P) \log \sqrt{\frac{2 P}{\pi e}}+H_{e}(\beta(P)), \quad P \leq 6.303 \mathrm{~dB}
$$

where $\beta(P)=1 / 2-Q(2 \sqrt{P})$ and $H_{e}(x)=-x \log (x)-(1-$ $x) \log (x)$ is the binary entropy function with the units of nats.

The bounds are plotted in Fig. 2, where the lower bound is taken from [7] with optimized input distribution. The refined bound is best for SNR from 0 to $5 \mathrm{~dB}$ but is valid only for SNR below $6.3 \mathrm{~dB}$.

\section{Complex AWGN Channel}

Consider next the complex-alphabet AWGN channel

$$
Y=X+Z
$$

where $Z=Z_{R}+j Z_{I}, j=\sqrt{-1}$, and $Z_{R}, Z_{I}$ are independent Gaussian random variables with mean 0 and variance 1 . The channel density in Cartesian coordinates with $x=\left[x_{R}, x_{I}\right]$ and $y=\left[y_{R}, y_{I}\right]$ is

$$
p_{Y \mid X}(y \mid x)=\frac{1}{2 \pi} e^{-\|y-x\|^{2} / 2} .
$$

In spherical coordinates with $x=\left[|x|, \phi_{x}\right]$ and $y=\left[|y|, \phi_{y}\right]$, the density is

$$
p_{Y \mid X}(y \mid x)=\frac{1}{2 \pi} e^{-\left(|y|^{2}+|x|^{2}-2|x||y| \cos \left(\phi_{y}-\phi_{x}\right)\right) / 2} .
$$

Consider again the peak power constraint $|X| \leq A$ where $A>0$. We choose the test density

$$
q_{Y}(y)= \begin{cases}\frac{\beta}{\pi A^{2}}, & |y| \leq A \\ \frac{1-\beta}{2 \pi(1+\sqrt{\pi / 2} A)} e^{-(|y|-A)^{2} / 2}, & |y|>A .\end{cases}
$$

Again, the test density is uniform in the interval $|y| \leq A$ and is a "split and scaled" Gaussian density for $|y|>A$. Inserting into the divergence (5), we have1

$$
\begin{aligned}
& D\left(p_{Y \mid X}(\cdot \mid x) \| q_{Y}(\cdot)\right)=-\log (2 \pi e)-\mathrm{E}\left[\log q_{Y}(Y)\right] \\
& =\log \frac{\pi A^{2}}{2 \pi e \beta}-\mathrm{E}\left[\log \left(q_{Y}(Y) \frac{\pi A^{2}}{\beta}\right)\right] \\
& =\log \frac{A^{2}}{2 e \beta}-\int_{A}^{\infty} e^{-\left(z^{2}+|x|^{2}\right) / 2} I_{0}(z|x|) \\
& {\left[\log \left(\frac{(1-\beta) \pi A^{2}}{2 \pi(1+\sqrt{\pi / 2} A) \beta}\right)-\frac{(z-A)^{2}}{2}\right] z d z .} \\
& =\log \frac{A^{2}}{2 e \beta}+\log \left(\frac{2 e(1+\sqrt{\pi / 2} A) \beta}{(1-\beta) A^{2}}\right) Q_{1}(|x|, A) \\
& -\tilde{g}(|x|, A)
\end{aligned}
$$

where

$$
\tilde{g}(|x|, A)=\int_{A}^{\infty} e^{-\left(z^{2}+|x|^{2}\right) / 2} I_{0}(z|x|)\left[1-\frac{(z-A)^{2}}{2}\right] z d z .
$$

Lemma 1: $\tilde{g}(|x|, A)$ in (24) is positive for $|x| \in[0, A]$.

Proof: We use the definition of $I_{0}(x)$ to re-write (24) as

$$
\begin{aligned}
& \frac{1}{\sqrt{2 \pi}} \int_{0}^{\pi} e^{-\left(|x|^{2} / 2\right) \sin ^{2} \phi} \\
& {\left[\int_{A}^{\infty}\left[2-(z-A)^{2}\right] z \psi(z-|x| \cos \phi) d z\right] d \phi .}
\end{aligned}
$$

The integral in square brackets can be simplified by substituting $\tilde{z}=z-|x| \cos \phi$ and $u=A-|x| \cos \phi$ to become

$$
\int_{u}^{\infty}\left[2-(\tilde{z}-u)^{2}\right](\tilde{z}-u+A) \psi(\tilde{z}) d \tilde{z} .
$$

Using (2)-(4), the integral (26) evaluates to

$$
u(A-u)[\psi(u)-u Q(u)]+(A+u) Q(u)
$$

or alternatively to

$$
-(A-u)\left[\left(1+u^{2}\right) Q(u)-u \psi(u)\right]+2 A Q(u) .
$$

Note that we have $0 \leq u \leq A+|x|$. We consider two cases.

- $0 \leq u \leq A$ : We have $u(A-u) \geq 0$ and the bound on the right-hand side of (1) tells us that (27) is negative.

- $A \leq u \leq A+|x|$ : We have $A-u \leq 0$ and the bound on the left-hand side of (1) tells us that (28) is positive.

Thus, the expression (27) (or equivalently (28)) is positive. But this implies that the integrals in (25)-(26) are all positive, and we conclude that $\tilde{g}(A, x)$ is positive.

\section{A. McKellips-type Bound}

Using Lemma 1 in (23), we have

$$
\begin{aligned}
& D\left(p_{Y \mid X}(\cdot \mid x) \| q_{Y}(\cdot)\right) \\
& \leq \log \frac{A^{2}}{2 e \beta}+\log \left(\frac{2 e(1+\sqrt{\pi / 2} A) \beta}{(1-\beta) A^{2}}\right) Q_{1}(|x|, A) .
\end{aligned}
$$

${ }^{1}$ By symmetry we may restrict attention to $\phi_{x}=0$, i.e., real $x$ satisfying $0 \leq x \leq A$. 
By choosing $\beta$ to make the second term above zero, we have

$$
\beta=\frac{A^{2}}{A^{2}+2 e(1+\sqrt{\pi / 2} A)}
$$

which results in the McKellips-type bound

$$
D\left(p_{Y \mid X}(\cdot \mid x) \| q_{Y}(\cdot)\right) \leq \log \left(1+\sqrt{\pi / 2} A+\frac{A^{2}}{2 e}\right) .
$$

Since this bound is independent of $x$, we have

$$
C<\log \left(1+\sqrt{\pi / 2} A+\frac{A^{2}}{2 e}\right) .
$$

We combine (32) with the capacity under the (weaker) average power constraint $\mathrm{E}\left[|X|^{2}\right] \leq A^{2}$. Observe that the complex noise has power 2 so the corresponding SNR is $P=A^{2} / 2$. We thus have the simple bound

$$
C \leq \min \left\{\log \left(1+\sqrt{\pi P}+\frac{P}{e}\right), \log (1+P)\right\}
$$

where we measure the rate per complex symbol (two real dimensions).

The high-SNR power loss is $10 \log _{10}(e) \approx 4.34 \mathrm{~dB}$. Again, however, this comparison is based on equating the maximum power $P$ with the average power. Instead, if we use the uniform distribution for $X$ then the average power is $P / 2$ and the high-SNR power loss reduces to a shaping loss of $10 \log _{10}(e / 2) \approx 1.33 \mathrm{~dB}$.

\section{B. Refined Bound}

We refine the upper bound for the complex AWGN channel in a manner similar to the refinement in the real AWGN case. First, rewrite the final expression for $D\left(p_{Y \mid X}(\cdot \mid x) \| q_{Y}(\cdot)\right)$ in (22) as

$$
\begin{aligned}
& D\left(p_{Y \mid X}(\cdot \mid x) \| q_{Y}(\cdot)\right)=\log \left(\frac{A^{2}}{2 e \beta}\right) \\
& +\log \frac{2 \beta(1+\sqrt{\pi / 2} A)}{(1-\beta) A^{2}} Q_{1}(|x|, A)+g(|x|, A)
\end{aligned}
$$

where

$$
g(|x|, A)=\int_{A}^{\infty} \frac{(z-A)^{2}}{2} z e^{-\left(z^{2}+|x|^{2}\right) / 2} I_{0}(z|x|) d z .
$$

The functions $Q_{1}(|x|, A)$ and $g(|x|, A)$ are both increasing in $|x|$. This is proved as part of the general $n$-dimensional case in Appendix B. Hence, for a positive $\log \frac{2 \beta(1+\sqrt{\pi / 2} A)}{(1-\beta) A^{2}}$ the expression (34) is maximized at $|x|=A$, and we obtain

$$
\begin{aligned}
& D\left(p_{Y \mid X}(\cdot \mid x) \| q_{Y}(\cdot)\right) \leq \log \left(\frac{A^{2}}{2 e \beta}\right) \\
& +\log \frac{2 \beta(1+\sqrt{\pi / 2} A)}{(1-\beta) A^{2}} Q_{1}(A, A)+g(A, A)
\end{aligned}
$$

provided that

$$
\beta \geq \frac{A^{2}}{A^{2}+2(1+\sqrt{\pi / 2} A)} .
$$

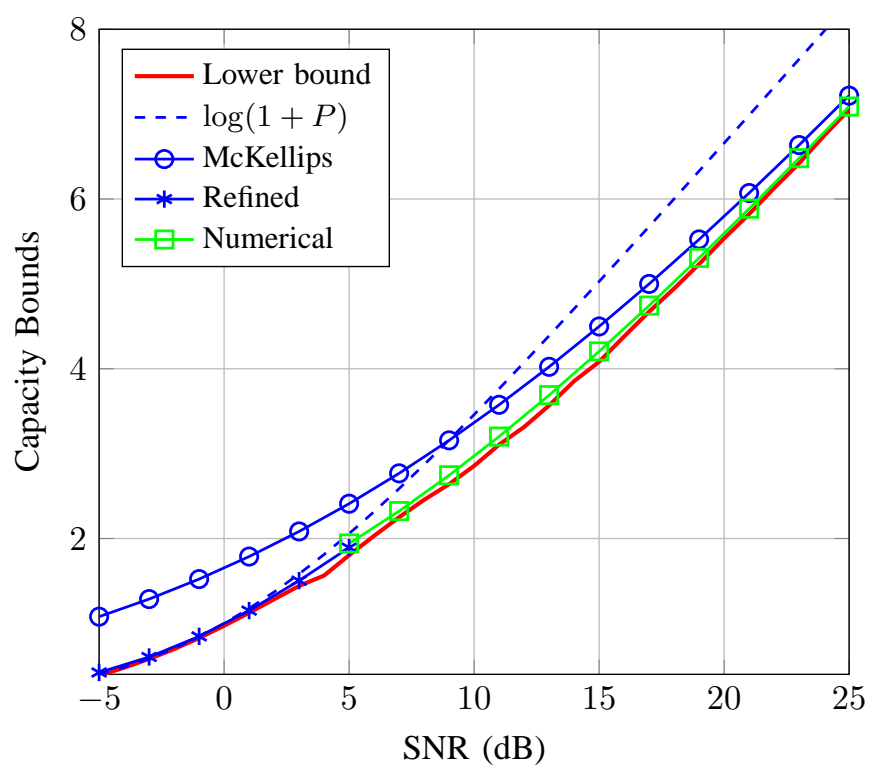

Fig. 3. Capacity bounds for complex AWGN channels. The rate units are bits per 2 dimensions.

Rewriting the bound of (35) as

$$
\begin{aligned}
& D\left(p_{Y \mid X}(\cdot \mid x) \| q_{Y}(\cdot)\right) \leq Q_{1}(A, A) \log \frac{1+\sqrt{\pi / 2} A}{e} \\
& +\left(1-Q_{1}(A, A)\right) \log \frac{A^{2}}{2 e}+g(A, A) \\
& -\left(1-Q_{1}(A, A)\right) \log \beta-Q_{1}(A, A) \log (1-\beta)
\end{aligned}
$$

we see that $\beta=1-Q_{1}(A, A)$ minimizes the RHS of (37). However, from (36) this choice of $\beta$ is valid only if

$$
1-Q_{1}(A, A) \geq \frac{A^{2}}{A^{2}+2(1+\sqrt{\pi / 2} A)}
$$

which requires $A<2.36$ numerically. Therefore, setting $P=$ $A^{2} / 2$ we have

$$
\begin{aligned}
C \leq & (1-\beta(P)) \log (1+\sqrt{\pi P})+\beta(P) \log \frac{P}{e} \\
& +H_{e}(\beta(P))-\tilde{g}(\sqrt{2 P}, \sqrt{2 P}), \quad P \leq 4.45 \mathrm{~dB}
\end{aligned}
$$

where $\beta(P)=1-Q_{1}(\sqrt{2 P}, \sqrt{2 P})$ and we have used the relationship $\tilde{g}(|x|, A)=Q_{1}(|x|, A)-g(|x|, A)$.

The bounds are plotted in Fig. 3. The lower bound is obtained by evaluating mutual information for the equi-probable complex constellation

$$
\{0\} \cup \bigcup_{0 \leq k \leq\lfloor A / 2\rfloor-1}\left\{(A-2 k) e^{j n \theta_{k}}: 0 \leq n \leq N_{k}\right\}
$$

where $\theta_{k}=\frac{2 \pi}{3(A-2 k)}$ and $N_{k}=\lfloor 3(A-2 k)\rfloor$. We see that the refined upper bound, valid for SNR less than $4.45 \mathrm{~dB}$, is close to the lower bound. The numerical evaluation of $\min _{\beta} \max _{x} D\left(p_{Y \mid X}(\cdot \mid x) \| q_{Y}(\cdot)\right)$ is seen to yield best bounds throughout. 


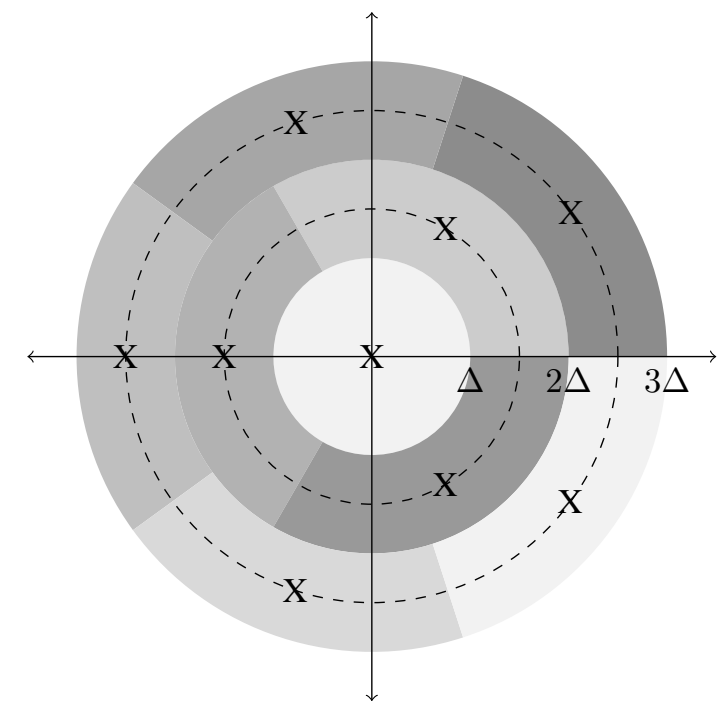

Fig. 4. The two-dimensional constellation $A_{3}$. X denotes a constellation point. For $x \in A_{3},(X+U) \mid X=x$ is distributed uniformly in the shaded region around $x$.

\section{Analytical lower bound}

A lower bound on the capacity of real AWGN channels was derived in [12] by using PAM-like constellations. The input was peak-power constrained. By selecting the number of points suitably, PAM achieves rates within a small gap from the average-power constrained capacity $\frac{1}{2} \log (1+\mathrm{SNR})$.

For two-dimensions, consider the constellation

$$
A_{N}=\{0\} \cup \bigcup_{n=1}^{N-1}\left\{(n+0.5) \Delta e^{j(l+0.5) \theta_{n}}: l=0,1, \ldots, 2 n\right\}
$$

where $N \geq 2$ is a positive integer, $\Delta$ is a positive real number and $\theta_{n}=2 \pi /(2 n+1)$. The set $A_{N}$ contains $N^{2}$ points including the origin and $(2 n+1)$ equally-spaced points on a circle of radius $(n+0.5) \Delta$ for $n=1,2, \ldots, N-1$. The 9 points of $A_{3}$ are shown in Fig. 4 for illustration.

Define a random variable $U$ jointly distributed with $X \sim$ $\operatorname{Unif}\left(A_{N}\right)$ such that $\tilde{X}=X+U$ is uniformly distributed in the circle of radius $N \Delta$ around the origin. For the constellation $A_{3}$, the distribution of $\tilde{X} \mid X=x$ is illustrated through the shading around each point $x \in A_{3}$ in Fig. 4 Specifically, for the constellation $A_{N}, U$ is defined so that $(X+U) \mid X=0$ is uniform in the circle of radius $\Delta$ around the origin, and $(X+U) \mid X=(n+0.5) \Delta e^{j(l+0.5) \theta_{n}}$ is uniform in the region

$$
\left\{(r \cos \theta, r \sin \theta): n \leq \frac{r}{\Delta} \leq n+1, l \leq \frac{\theta}{\theta_{n}} \leq l+1\right\} .
$$

As before, the received value is $Y=X+Z$. Since $\tilde{X}-X-Y$ forms a Markov chain, we have $I(X ; Y) \geq I(\tilde{X} ; Y)$ by the data processing inequality. We further lower bound $I(\tilde{X} ; Y)$ by using a strategy similar to the real case in [12]. However, unlike the real case, $U$ and $X$ are correlated resulting in additional computations.

Since $I(\tilde{X} ; Y)=h(\tilde{X})-h(\tilde{X} \mid Y)$ and $h(\tilde{X})=$ $\log _{2} \pi N^{2} \Delta^{2}$, we lower bound $I(\tilde{X} ; Y)$ by first upper bound- ing $h(\tilde{X} \mid Y)$ as follows:

$$
\begin{aligned}
h(\tilde{X} \mid Y=y) & =-\int p(\tilde{x} \mid y) \log _{2} p(\tilde{x} \mid y) d \tilde{x} \\
& \leq-\int p(\tilde{x} \mid y) \log _{2} q_{y}(\tilde{x}) d \tilde{x}
\end{aligned}
$$

where $q_{y}(\tilde{x})$ is any valid density parametrized by $y$. Choosing $q_{y}(\tilde{x})=\frac{1}{2 \pi s^{2}} e^{-\|\tilde{x}-k y\|^{2} / 2 s^{2}}$ (with parameters $k$ and $s$ to be optimized later), we obtain

$$
\begin{gathered}
\frac{h(\tilde{X} \mid Y=y)}{\log _{2} e} \leq \log 2 \pi s^{2}+\frac{1}{2 s^{2}} E\left[\|\tilde{X}-k y\|^{2} \mid Y=y\right] \\
\frac{h(\tilde{X} \mid Y)}{\log _{2} e}=\log 2 \pi s^{2}+\frac{1}{2 s^{2}} E\left[\|\tilde{X}-k Y\|^{2}\right] .
\end{gathered}
$$

Expanding using $\tilde{X}=X+U$ and $Y=X+Z$, and using the fact that $Z$ is independent of $X$ and $U$, we have

$$
E\left[\|\tilde{X}-k Y\|^{2}\right]=\frac{N^{2} \Delta^{2}}{2}-2\left(1+\rho_{N}\right) P_{N} k+\left(P_{N}+2\right) k^{2}
$$

where $P_{N} \triangleq E\left[X^{2}\right], \rho_{N} \triangleq \operatorname{Re}\left(E\left[X^{*} U\right]\right) / P_{N}$, and we have used $E\left[\|\tilde{X}\|^{2}\right]=\frac{N^{2} \Delta^{2}}{2}$. To obtain the lowest upper bound for $E\left[\|\tilde{X}-k Y\|^{2}\right]$, we set $k=k^{*}=\frac{\left(1+\rho_{N}\right) P_{N}}{2+P_{N}}$ and $s^{2}=$ $\frac{1}{2} E\left[\left\|\tilde{X}-k^{*} Y\right\|^{2}\right]$. Simplifying, we have

$$
h(\tilde{X} \mid Y) \leq \log _{2}\left(\pi e\left[\frac{N^{2} \Delta^{2}}{2}-\frac{P_{N}^{2}\left(1+\rho_{N}\right)^{2}}{P_{N}+2}\right]\right) .
$$

We continue to bound lower $I(\tilde{X} ; Y)$ by

$$
\begin{aligned}
& \log _{2} \pi N^{2} \Delta^{2}-\log _{2}\left(\pi e\left[\frac{N^{2} \Delta^{2}}{2}-\frac{P_{N}^{2}\left(1+\rho_{N}\right)^{2}}{P_{N}+2}\right]\right) \\
& =\log _{2} N^{2}-\log _{2} \frac{e}{2}-\log _{2}\left(N^{2}-\frac{P_{N}^{2}\left(1+\rho_{N}\right)^{2}}{\left(1+P_{N} / 2\right) \Delta^{2}}\right) .
\end{aligned}
$$

Defining $\tilde{C}=\log _{2}\left(1+P_{N} / 2\right)$, and setting $N^{2}=\alpha 2^{\tilde{C}}=$ $\alpha\left(1+P_{N} / 2\right)$ and simplifying, we have

$$
I(\tilde{X} ; Y) \geq \tilde{C}-\log _{2} \frac{e}{2}-\log _{2}\left(\frac{N^{2}}{\alpha}-\frac{P_{N}^{2}\left(1+\rho_{N}\right)^{2}}{N^{2} \Delta^{2}}\right) .
$$

In Appendix $\mathrm{C}$, we show that $P_{N}=\frac{\Delta^{2} N^{2}}{2}\left(1-O\left(1 / N^{2}\right)\right)$, $-0.66 \leq \rho_{N} N^{2} /\left(1-O\left(1 / N^{2}\right)\right) \leq-0.64$ and provide details of the simplification needed to obtain the following lower bound:

$$
I(X ; Y) \geq \tilde{C}-0.45-\log _{2}\left(1+\frac{1.82}{\alpha}+O\left(\frac{1}{N^{2}}\right)\right) .
$$

We see that the gap to the average-power constrained capacity with a finite constellation can be made as small as 0.45 by choosing a large enough $\alpha$ at high rates (large $N$ ). For moderate $N$, choosing $\alpha=4$ results in a gap of less than 1 to capacity. Finally, the rate in (47) is achieved at a peakpower constraint of $|X| \leq(N-0.5) \Delta$ or an equivalent $\mathrm{SNR}=(N-0.5)^{2} \Delta^{2} / 2$. This lets one compare the analytical lower bound against the other bounds shown in Fig. 3 . 


\section{V. $n$-DIMENSIONAL AWGN CHANNEL}

Consider next the $n$-dimensinal AWGN channel

$$
\underline{Y}=\underline{X}+\underline{Z}
$$

where $\underline{Z}=\left[Z_{1}, Z_{2}, \ldots, Z_{n}\right]$ has independent Gaussian entries with mean 0 and variance 1 . The channel density in Cartesian coordinates with $\underline{x}=\left[x_{1}, \ldots, x_{n}\right]$ and $\underline{y}=\left[y_{1}, \ldots, y_{n}\right]$ is

$$
p_{\underline{Y} \mid \underline{X}}(\underline{y} \mid \underline{x})=\frac{1}{(2 \pi)^{n / 2}} e^{-\|\underline{y}-\underline{x}\|^{2} / 2} .
$$

The $n$-dimensional spherical coordinate system has a radial coordinate $r$ and $n-1$ angular coordinates $\phi_{i}, i=1,2, \ldots, n-$ 1 , where the domain of $\phi_{i}, i=1,2, \ldots, n-2$, is $[0, \pi)$, and the domain of $\phi_{n-1}$ is $[0,2 \pi) \underline{2}$ For a point $\underline{x}$ with spherical coordinates $\underline{x}=\left[r_{x}, \phi_{x, 1}, \ldots, \phi_{x, n-1}\right]$ we can compute the Cartesian coordinates $\underline{x}=\left[x_{1}, \ldots, x_{n}\right]$ via

$$
\begin{aligned}
x_{1} & =r_{x} \cos \left(\phi_{x, 1}\right) \\
x_{2} & =r_{x} \sin \left(\phi_{x, 1}\right) \cos \left(\phi_{x, 2}\right) \\
x_{3} & =r_{x} \sin \left(\phi_{x, 1}\right) \sin \left(\phi_{x, 2}\right) \cos \left(\phi_{x, 3}\right) \\
\vdots & \\
x_{n-1} & =r_{x} \sin \left(\phi_{x, 1}\right) \ldots \sin \left(\phi_{x, n-2}\right) \cos \left(\phi_{x, n-1}\right) \\
x_{n} & =r_{x} \sin \left(\phi_{x, 1}\right) \ldots \sin \left(\phi_{x, n-2}\right) \sin \left(\phi_{x, n-1}\right) .
\end{aligned}
$$

In spherical coordinates the channel density has a complex form due to the many sine and cosine terms. However, by symmetry we may restrict attention to points $\underline{x}$ with $\phi_{x, i}=0$ for all $i$. For such $\underline{x}$, the channel density in $n$-dimensional spherical coordinates is simply

$$
p_{\underline{Y} \mid \underline{X}}(\underline{y} \mid \underline{x})=\frac{1}{(2 \pi)^{n / 2}} e^{-\left(r_{y}^{2}+r_{x}^{2}-2 r_{x} r_{y} \cos \phi_{y, 1}\right) / 2} .
$$

Consider now the $n$-dimensional amplitude constraint $\|\underline{X}\| \leq A$ where $A>0$. We choose the test density

$$
q_{\underline{Y}}(\underline{y})= \begin{cases}\frac{\beta}{\operatorname{Vol}(A)}, & r_{y} \leq A \\ \frac{1-\beta}{k_{n}(A)(2 \pi)^{n / 2}} e^{-\left(r_{y}-A\right)^{2} / 2}, & r_{y}>A\end{cases}
$$

where $\operatorname{Vol}(r)$ is the volume of an $n$-dimensional ball with radius $r$ and $k_{n}(A)$ is a constant that ensures that $q_{\underline{Y}}(\cdot)$ is a density. Again, the test density is uniform in the interval $r_{y} \leq$ $A$ and is a "split and scaled" Gaussian density for $r_{y}>A$. We have 3

$$
\operatorname{Vol}(r)=\frac{\pi^{n / 2}}{\Gamma(n / 2+1)} r^{n}
$$

where $\Gamma(\cdot)$ is Euler's gamma function. To compute $k_{n}(A)$, observe that we require

$$
\frac{1}{k_{n}(A)(2 \pi)^{n / 2}} \int_{A}^{\infty} \int_{0}^{\pi} \cdots \int_{0}^{\pi} \int_{0}^{2 \pi} e^{-(r-A)^{2} / 2} d V=1
$$

where

$$
d V=r^{n-1} d r\left[\prod_{i=1}^{n-2} \sin ^{n-1-i}\left(\phi_{i}\right) d \phi_{i}\right] d \phi_{n-1}
$$

${ }^{2}$ See http://en.wikipedia.org/wiki/N-sphere

${ }^{3}$ See http://en.wikipedia.org/wiki/Volume_of_an_n-ball . is the spherical volume element in $n$ dimensions. We thus have

$$
\begin{aligned}
& k_{n}(A)=\frac{2}{2^{n / 2} \Gamma\left(\frac{n}{2}\right)} \int_{A}^{\infty} e^{-(r-A)^{2} / 2} r^{n-1} d r \\
& =\frac{2^{\frac{2-n}{2}}}{\Gamma\left(\frac{n}{2}\right)} \int_{A}^{\infty} e^{\frac{-(r-A)^{2}}{2}}\left(\sum_{i=0}^{n-1}\left(\begin{array}{c}
n-1 \\
i
\end{array}\right) A^{n-1-i}(r-A)^{i}\right) d r \\
& =\frac{2^{\frac{2-n}{2}}}{\Gamma\left(\frac{n}{2}\right)}\left[\sum_{i=0}^{n-1}\left(\begin{array}{c}
n-1 \\
i
\end{array}\right) A^{n-1-i} \int_{0}^{\infty} r^{i} e^{-r^{2} / 2} d r\right] .
\end{aligned}
$$

Using the standard integral

$$
\int_{0}^{\infty} x^{n} e^{-a x^{2}} d x=\frac{\Gamma\left(\frac{n+1}{2}\right)}{2 a^{\frac{n+1}{2}}}
$$

the expression for $k_{n}(A)$ in 57) simplifies to

$$
k_{n}(A)=\sum_{i=0}^{n-1}\left(\begin{array}{c}
n-1 \\
i
\end{array}\right) \frac{\Gamma\left(\frac{n-i}{2}\right)}{2^{i / 2} \Gamma\left(\frac{n}{2}\right)} A^{i} .
$$

For example, for $n=1$ we have $k_{1}=1$, and for $n=2$ we have $k_{2}=1+\sqrt{\pi / 2} A$.

\section{A. McKellips-type Bound}

The divergence in (5) can be written as

$$
\begin{aligned}
& D\left(p_{\underline{Y} \mid \underline{X}}(\cdot \mid \underline{x}) \| q_{\underline{Y}}(\cdot)\right)=-\frac{n}{2} \log (2 \pi e)-\mathrm{E}\left[\log q_{\underline{Y}}(\underline{Y})\right] \\
& =\log \frac{\operatorname{Vol}(\mathrm{A})}{(2 \pi e)^{n / 2} \beta}-\mathrm{E}\left[\log \left(q_{\underline{Y}}(\underline{Y}) \frac{\operatorname{Vol}(A)}{\beta}\right)\right] .
\end{aligned}
$$

The expectation in the above equation can be simplified and written as

$$
\begin{aligned}
\frac{2}{2^{\frac{n-1}{2}} \Gamma\left(\frac{n-1}{2}\right)} \int_{0}^{\pi} \sin ^{n-2}\left(\phi_{1}\right) e^{-\left(r_{x}^{2} / 2\right) \sin ^{2} \phi_{1}} \\
\quad\left\{\int_{A}^{\infty} \psi\left(z-r_{x} \cos \phi_{1}\right)\right. \\
\left.\quad\left[\log \frac{(1-\beta) \operatorname{Vol}(A)}{(2 \pi)^{n / 2} \beta k_{n}(A)}-\frac{\left(r_{y}-A\right)^{2}}{2}\right] r_{y}^{n-1} d r_{y}\right\} d \phi_{1} .
\end{aligned}
$$

For $n \geq 2$, define the functions

$$
\begin{aligned}
& \tilde{I}_{n}(x)=\frac{2}{2^{\frac{n-1}{2}} \Gamma\left(\frac{n-1}{2}\right) \sqrt{2 \pi}} \int_{0}^{\pi} e^{x \cos \phi}(\sin \phi)^{n-2} d \phi \\
& Q_{n}(x, A)=\int_{A}^{\infty} e^{-\left(z^{2}+x^{2}\right) / 2} \tilde{I}_{n}(z x) z^{n-1} d z \\
& g_{n}(x, A)=\int_{A}^{\infty} \frac{(z-A)^{2}}{2} e^{-\left(z^{2}+x^{2}\right) / 2} \tilde{I}_{n}(z x) z^{n-1} d z \\
& \tilde{g}_{n}(x, A)=\int_{A}^{\infty}\left(\frac{n}{2}-\frac{(z-A)^{2}}{2}\right) e^{-\left(z^{2}+x^{2}\right) / 2} \tilde{I}_{n}(z x) z^{n-1} d z
\end{aligned}
$$

In terms of the above functions, we can write

$$
\begin{aligned}
& D\left(p_{\underline{Y} \mid \underline{X}}(\cdot \mid \underline{x}) \| q_{\underline{Y}}(\cdot)\right)=\log \frac{\operatorname{Vol}(\mathrm{A})}{(2 \pi e)^{n / 2} \beta} \\
& +\left(\log \frac{(2 \pi e)^{n / 2} \beta k_{n}(A)}{(1-\beta) \operatorname{Vol}(A)}\right) Q_{n}(x, A)-\tilde{g}_{n}(x, A) .
\end{aligned}
$$


In Appendix A, we show that $\tilde{g}_{n}(x, A)$ is positive. We make the second $\log$ term in 65 zero by choosing

$$
\beta=\frac{\operatorname{Vol}(A)}{\operatorname{Vol}(A)+(2 \pi e)^{n / 2} k_{n}(A)}
$$

and we obtain the bound

$$
C \leq \log \left(k_{n}(A)+\frac{\operatorname{Vol}(A)}{(2 \pi e)^{n / 2}}\right) .
$$

We combine this result with the capacity under the (weaker) average power constraint $\mathrm{E}\left[\|X\|^{2}\right] \leq A^{2}$. Observe that the complex noise has power $n$ so the corresponding SNR is $P=$ $A^{2} / n$. We thus have the bound

$C \leq \min \left\{\log \left(k_{n}(\sqrt{n P})+\frac{\operatorname{Vol}(\sqrt{n P})}{(2 \pi e)^{n / 2}}\right), \frac{n}{2} \log (1+P)\right\}$

where we measure the rate per $n$-dimensional symbol.

\section{B. Refined Bound}

The refinement is similar to the 2-dimensional case. We rewrite $D\left(p_{\underline{Y} \mid \underline{X}}(\cdot \mid \underline{x}) \| q_{\underline{Y}}(\cdot)\right)$ in (65) as follows:

$$
\begin{aligned}
& D\left(p_{\underline{Y} \mid \underline{X}}(\cdot \mid \underline{x}) \| q_{\underline{Y}}(\cdot)\right)=\log \frac{\operatorname{Vol}(A)}{(2 \pi e)^{n / 2} \beta} \\
& +\left(\log \frac{(2 \pi)^{n / 2} k_{n}(A) \beta}{(1-\beta) \operatorname{Vol}(A)}\right) Q_{n}(x, A)+g_{n}(x, A)
\end{aligned}
$$

where we have used the relationship $\tilde{g}_{n}(x, A)=\frac{n}{2} Q_{n}(x, A)-$ $g_{n}(x, A)$. As shown in Appendix B, the functions $Q_{n}(x, A)$ and $g_{n}(x, A)$ are both increasing in $x$. Hence, for a positive $\log \frac{(2 \pi)^{n / 2} k_{n}(A) \beta}{(1-\beta) \operatorname{Vol}(A)}$, the RHS of (68) is maximized at $x=A$, and we obtain the bound

$$
\begin{aligned}
& D\left(p_{\underline{Y} \mid \underline{X}}(\cdot \mid \underline{x}) \| q_{\underline{Y}}(\cdot)\right) \leq \log \frac{\operatorname{Vol}(\mathrm{A})}{(2 \pi e)^{n / 2} \beta} \\
& +\left(\log \frac{(2 \pi)^{n / 2} \beta k_{n}(A)}{(1-\beta) \operatorname{Vol}(A)}\right) Q_{n}(A, A)+g_{n}(A, A)
\end{aligned}
$$

provided that

$$
\beta \geq \frac{\operatorname{Vol}(A)}{(2 \pi)^{n / 2} k_{n}(A)+\operatorname{Vol}(A)} .
$$

Rewriting (69) as

$$
\begin{aligned}
& D\left(p_{\underline{Y} \mid \underline{X}}(\cdot \mid \underline{x}) \| q_{\underline{Y}}(\cdot)\right) \leq Q_{n}(A, A) \log \frac{k_{n}(A)}{e^{n / 2}} \\
& +\left(1-Q_{n}(A, A)\right) \log \frac{\operatorname{Vol}(A)}{(2 \pi e)^{n / 2}}+g_{n}(A, A) \\
& -\left(1-Q_{n}(A, A)\right) \log \beta-Q_{n}(A, A) \log (1-\beta)
\end{aligned}
$$

we see that $\beta=1-Q_{n}(A, A)$ minimizes the RHS of (71). However, from (70) this choice of $\beta$ is valid only if

$$
1-Q_{n}(A, A) \geq \frac{\operatorname{Vol}(A)}{(2 \pi)^{n / 2} k_{n}(A)+\operatorname{Vol}(A)}
$$

which requires $A<A_{n}^{*}$, where $A_{n}^{*}$ is the smallest positive value that results in equality in (72). The value $A_{n}^{*}$ can be determined numerically. Choosing $P=A^{2} / n$ and $P_{n}^{*}=$ $\left(A_{n}^{*}\right)^{2} / n$, we obtain the bound

$$
\begin{aligned}
C \leq & \left(1-\beta_{n}(P)\right) \log \left(k_{n}(\sqrt{n P})\right)+\beta_{n}(P) \log \frac{\operatorname{Vol}(\sqrt{n P})}{(2 \pi e)^{n / 2}} \\
& +H_{e}\left(\beta_{n}(P)\right)-\tilde{g}(\sqrt{n P}, \sqrt{n P}), \quad P \leq P_{n}^{*}
\end{aligned}
$$

where $\beta_{n}(P)=1-Q_{n}(\sqrt{n P}, \sqrt{n P})$.

\section{Volume-based Lower Bound}

To obtain a lower bound, we use the volume-based method introduced in [13]. The capacity of the $n$-dimensional AWGN channel $\underline{Y}=\underline{X}+\underline{Z}$ with the peak constraint $|\underline{X}| \leq A$ is

$$
C=\lim _{m \rightarrow \infty} \frac{1}{m} \sup _{p\left(\underline{x}^{m}\right) \in \mathcal{P}_{n}^{m}} I\left(\underline{X}^{m} ; \underline{Y}^{m}\right)
$$

where $\mathcal{P}_{n}^{m}$ is the set of all valid distributions satisfying the $n$-dimensional peak-power constraint. Now consider

$$
\begin{aligned}
\frac{1}{m} I\left(\underline{X}^{m} ; \underline{Y}^{m}\right) & =\frac{1}{m}\left(h\left(\underline{Y}^{m}\right)-h\left(\underline{Z}^{m}\right)\right) \\
& =\frac{1}{m} h\left(\underline{Y}^{m}\right)-\frac{n}{2} \log (2 \pi e) .
\end{aligned}
$$

Using the entropy-power inequality, we have

$$
\begin{aligned}
\sup _{p\left(\underline{x}^{m}\right) \in \mathcal{P}_{n}^{m}} e^{\frac{2}{m n} h\left(\underline{Y}^{m}\right)} & \geq \sup _{p\left(\underline{x}^{m}\right) \in \mathcal{P}_{n}^{m}} e^{\frac{2}{m n} h\left(\underline{X}^{m}\right)}+e^{\frac{2}{m n} h\left(\underline{Z}^{m}\right)} \\
& \geq e^{\frac{2}{m n} \log (\operatorname{Vol}(A))^{m}}+e^{\log (2 \pi e)} \\
& \geq\left(\operatorname{Vol}_{n}(A)\right)^{2 / n}+2 \pi e
\end{aligned}
$$

Therefore, we have

$$
\sup _{p\left(\underline{x}^{m}\right) \in \mathcal{P}_{n}^{m}} \frac{1}{m} h\left(\underline{Y}^{m}\right) \geq \frac{n}{2} \log \left((\operatorname{Vol}(A))^{2 / n}+2 \pi e\right) .
$$

Using (77) in (75), we have

$$
\begin{aligned}
C & \geq \frac{n}{2} \log \left(1+\frac{\operatorname{Vol}(A)^{2 / n}}{2 \pi e}\right) \\
& =\log \left(O\left(A^{n-2}\right)+\frac{\operatorname{Vol}(A)}{(2 \pi e)^{n / 2}}\right) .
\end{aligned}
$$

Comparing (78) with the McKellips-type upper bound in (67), we see that two bounds meet as SNR tends to infinity, and they differ by $O\left(A^{n-1}\right)$ inside the logarithm. Thus, for moderate SNR the McKellips-type bound could be improved. Comparing with the refined bound is not straight-forward, and a numerical comparison for $n=2$ is shown in Fig. 3. The case $n=4$ is interesting because coherent optical communication with two polarizations results in a 4-dimensional signal space. The bounds for $n=4$ are plotted in Fig. 5. As expected, the volume-based lower bound meets the McKellips-type bound at high SNR. The analytical refined bound is valid for SNR less than $P_{4}^{*} \approx 7.92 \mathrm{~dB}$. Numerical evaluation of $\min _{\beta} \max _{x} D\left(p_{\underline{Y} \mid \underline{X}}(\cdot \mid \underline{x}) \| q_{\underline{Y}}(\cdot)\right)$ is seen to be close to the lower bound for moderate SNR. For lower SNR, the volumebased lower bound is not expected to be tight. 


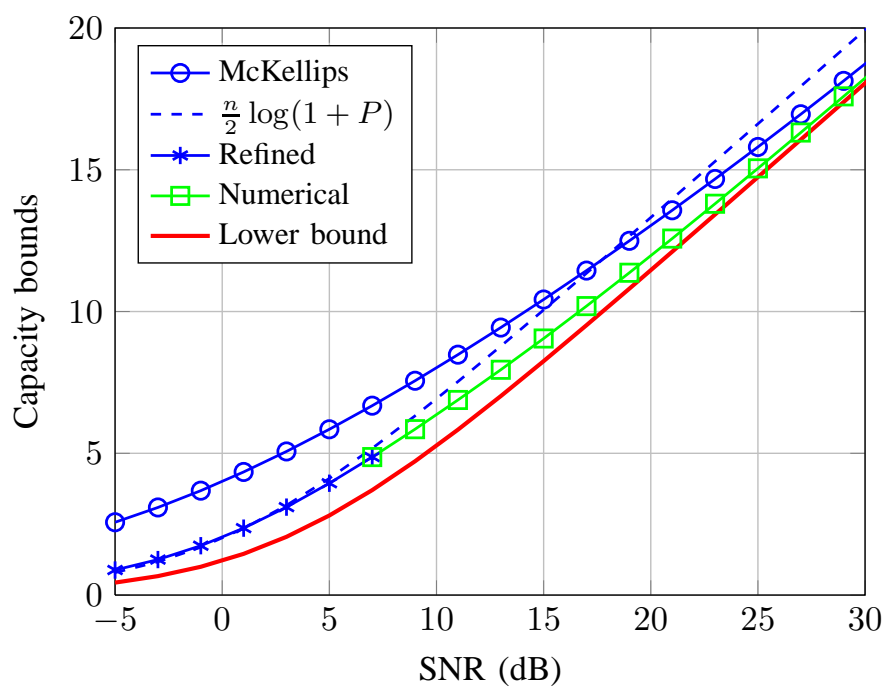

Fig. 5. Capacity bounds for 4-dimensional AWGN channels. The rate units are bits per 4 dimensions.

\section{Remarks}

Based on extensive numerical evaluations, we conjecture that the expression for $D\left(p_{\underline{Y} \mid \underline{X}}(\cdot \mid \underline{x}) \| q_{\underline{Y}}(\cdot)\right)$ in (68), denoted as

$$
\begin{aligned}
D_{n}(\beta, x) & \triangleq \log \frac{\operatorname{Vol}(A)}{(2 \pi e)^{n / 2} \beta}+g_{n}(x, A) \\
& +\left(\log \frac{(2 \pi)^{n / 2} k_{n}(A) \beta}{(1-\beta) \operatorname{Vol}(A)}\right) Q_{n}(x, A)
\end{aligned}
$$

is maximized over $x \in[0, A]$ (for a fixed $\beta$ ) at the endpoints $x=0$ or $x=A$. Under this conjecture, we have

$$
\min _{\beta} \max _{x} D_{n}(\beta, x)=\min _{\beta} \max \left(D_{n}(\beta, 0), D_{n}(\beta, A)\right) .
$$

Now, writing $D_{n}(\beta, x)$ as

$$
\begin{aligned}
D_{n}(\beta, x) & =-\left(1-Q_{n}(x, A)\right) \log \beta-Q_{n}(x, A) \log (1-\beta) \\
& + \text { terms independent of } \beta
\end{aligned}
$$

we see that, for a fixed $x$ and $\beta \in[0,1], D_{n}(\beta, x)$ achieves a minimum at $\hat{\beta}_{n}(x)=1-Q_{n}(x, A)$, decreases with $\beta$ for $\beta \in\left[0, \hat{\beta}_{n}(x)\right]$, and increases for $\beta \in\left[\hat{\beta}_{n}(x), 1\right]$. Let $\beta_{n}^{*}(A)$ be the value of $\beta$ for which $D_{n}(\beta, 0)=D_{n}(\beta, A)$.

Using the above, the minmax in (80) evaluates to

$$
\begin{gathered}
\min \left\{D_{n}\left(\beta_{n}^{*}(A), A\right), \max \left(D_{n}\left(\hat{\beta}_{n}(0), 0\right), D_{n}\left(\hat{\beta}_{n}(0), A\right)\right)\right. \\
\left.\max \left(D_{n}\left(\hat{\beta}_{n}(A), 0\right), D_{n}\left(\hat{\beta}_{n}(A), A\right)\right)\right\}
\end{gathered}
$$

To obtain an expression for $\beta_{n}^{*}(A)$, we simplify $D_{n}(\beta, 0)=$ $D_{n}(\beta, A)$ resulting in

$$
\beta_{n}^{*}(A)=\frac{\operatorname{Vol}(A)}{\operatorname{Vol}(A)+(2 \pi)^{n / 2} e^{c_{n}(A)} k_{n}(A)}
$$

where $c_{n}(A)=\frac{g_{n}(A, A)-g_{n}(0, A)}{Q_{n}(0, A)-Q_{n}(A, A)}$. Interestingly, as $A \rightarrow$ $\infty, c_{n}(A) \rightarrow-1 / 2$, and we see that $\beta_{n}^{*}(A)$ tends to the McKellips-type expression in (66).

Finally, we remark that the best upper bounds are as follows:
1) at low SNR: the average-power capacity $\frac{n}{2} \log (1+P)$;

2) at moderate SNR: the refined upper bound which evaluates to $D_{n}\left(\hat{\beta}_{n}(A), A\right)$

3) at high SNR: $D_{n}\left(\beta_{n}^{*}(A), A\right)$ which tends to the McKellips-type bound.

The exact range of low, moderate, and high SNR depends on the dimension $n$.

\section{ACKNOWLEDGMENT}

G. Kramer and G. Böcherer were supported by the German Federal Ministry of Education and Research in the framework of an Alexander von Humboldt Professorship.

\section{REFERENCES}

[1] J. G. Smith, "The information capacity of amplitude- and varianceconstrained scalar gaussian channels," Inf. and Control, vol. 18, no. 3 , pp. $203-219,1971$.

[2] S. Shamai and I. Bar-David, "The capacity of average and peak-powerlimited quadrature gaussian channels," IEEE Trans. Inf. Theory, vol. 41, no. 4, pp. 1060-1071, Jul 1995.

[3] A. Tchamkerten, "On the discreteness of capacity-achieving distributions," IEEE Trans. Inf. Theory, vol. 50, no. 11, pp. 2773-2778, Nov 2004.

[4] T. Chan, S. Hranilovic, and F. Kschischang, "Capacity-achieving probability measure for conditionally gaussian channels with bounded inputs," IEEE Trans. Inf. Theory, vol. 51, no. 6, pp. 2073-2088, June 2005.

[5] N. Sharma and S. Shamai, "Characterizing the discrete capacity achieving distribution with peak power constraint at the transition points," in IEEE Int. Symp. Inf. Theory and Its Appl., Dec 2008, pp. 1-6.

[6] Y. Polyanskiy and Y. Wu, "Peak-to-average power ratio of good codes for gaussian channel," IEEE Trans. Inf. Theory, vol. 60, no. 12, pp. 7655-7660, Dec 2014.

[7] A. McKellips, "Simple tight bounds on capacity for the peak-limited discrete-time channel," in IEEE Int. Symp. Inf. Theory, 2004, pp. 348348.

[8] I. Csiszár and J. Körner, Information Theory: Coding Theorems for Discrete Memoryless Systems. Cambridge University Press, 2011.

[9] A. Lapidoth and S. M. Moser, "Capacity bounds via duality with applications to multiple-antenna systems on flat fading channels," IEEE Trans. Inf. Theory, vol. 49, no. 10, pp. 2426-2467, Oct. 2003.

[10] A. Lapidoth, S. Moser, and M. Wigger, "On the capacity of free-space optical intensity channels," IEEE Trans. Inf. Theory, vol. 55, no. 10, pp. 4449-4461, Oct 2009.

[11] T. H. Cormen, C. Stein, R. L. Rivest, and C. E. Leiserson, Introduction to Algorithms, 2nd ed. McGraw-Hill Higher Education, 2001.

[12] L. H. Ozarow and A. D. Wyner, "On the capacity of the Gaussian channel with a finite number of input levels," IEEE Trans. Inf. Theory, vol. 36, no. 6, pp. 1426-1428, Nov. 1990.

[13] V. Jog and V. Anantharam, "An energy harvesting AWGN channel with a finite battery," in IEEE Int. Symp. Inf. Theory, June 2014, pp. 806-810.

\section{APPENDIX A}

We show that the function $\tilde{g}_{n}(x, A)$ in (64) is positive. First, we rewrite $\tilde{g}_{n}(x, A)$ as

$$
\begin{gathered}
\tilde{g}_{n}(x, A)=\frac{1}{2^{\frac{n-1}{2}} \Gamma\left(\frac{n-1}{2}\right)} \int_{0}^{\pi} \sin ^{n-2}(\phi) e^{-\left(x^{2} / 2\right) \sin ^{2} \phi} \\
\left\{\int_{A}^{\infty}\left[n-(z-A)^{2}\right] \psi(z-x \cos \phi) z^{n-1} d z\right\} d \phi .
\end{gathered}
$$

Now it suffices to show that the integral over $z$ above is positive. We set $\tilde{z}=z-x \cos \phi, u=A-x \cos \phi$ and simplify the integral as

$$
\int_{u}^{\infty}\left[n-(\tilde{z}-u)^{2}\right](\tilde{z}-u+A)^{n-1} \psi(\tilde{z}) d \tilde{z} .
$$


For example, for $n=2$ we recover (26). Now (85) can be rewritten as

$$
\sum_{i=0}^{n-1}\left(\begin{array}{c}
n-1 \\
i
\end{array}\right) A^{n-1-i} \int_{u}^{\infty}\left[n(\tilde{z}-u)^{i}-(\tilde{z}-u)^{i+2}\right] \psi(\tilde{z}) d \tilde{z}
$$

We claim that the integral inside the summation above is positive. In fact, we prove the following stronger inequality for $u \geq 0$ and a non-negative integer $i$ :

$$
\int_{u}^{\infty}\left[(i+1)(z-u)^{i}-(z-u)^{i+2}\right] \psi(z) d z \geq 0 .
$$

Since $n \geq i+1$ in 86 , the inequality (87) implies that the integral in 86 is positive. A proof of 87 is as follows:

$$
\begin{gathered}
\int_{u}^{\infty}(z-u)^{i} \psi(z) d z=\int_{u}^{\infty}(z-u)^{i} \psi(z)(z-u)^{\prime} d z \\
\left.\stackrel{(a)}{=}(z-u)^{i+1} \psi(z)\right|_{u} ^{\infty}-\int_{u}^{\infty}(z-u)\left[(z-u)^{i}(-z \psi(z))\right. \\
\left.+i(z-u)^{i-1} \psi(z)\right] d z \\
=\int_{u}^{\infty}(z-u)^{i+2} \psi(z) d z+u \int_{u}^{\infty}(z-u)^{i+1} \psi(z) d z \\
-i \int_{u}^{\infty}(z-u)^{i} \psi(z) d z
\end{gathered}
$$

where $(a)$ uses integration by parts. The above simplifies to (87) because $u \int_{u}^{\infty}(z-u)^{i+1} \psi(z) d z \geq 0$.

\section{APPENDIX B}

We use the double factorial notation

$$
n ! != \begin{cases}2 \cdot 4 \cdots n, & n: \text { even } \\ 1 \cdot 3 \cdots n, & n: \text { odd }\end{cases}
$$

Using $e^{x \cos \phi}=\sum_{m=0}^{\infty} \frac{(x \cos \phi)^{m}}{m !}$ in the integral of 61 , we get the Taylor series expansion for $\tilde{I}_{n}(x), n \geq 2$, as

$$
\tilde{I}_{n}(x)=\frac{2^{(2-n) / 2}}{\Gamma\left(\frac{n-1}{2}\right) \sqrt{\pi}} \sum_{m=0}^{\infty} a_{n-2, m} \frac{x^{m}}{m !}
$$

where $a_{n, m}=\int_{0}^{\pi} \sin ^{n} \phi \cos ^{m} \phi d \phi$. For odd $m$, it is easy to see that $a_{n, m}=0$. For even $m$, we have

$$
a_{n, m}= \begin{cases}\frac{\pi(m-1) ! !(n-1) ! !}{(n+m) ! !}, & n: \text { even } \\ \frac{2(m-1) ! !(n-1) ! !}{(n+m) ! !}, & n: \text { odd }\end{cases}
$$

Using the above $a_{m, n}$ in (88) and simplifying, we have

$$
\tilde{I}_{n}(x)=d_{n} \sum_{k=0}^{\infty} \frac{(2 k-1) ! !}{(n+2 k-2) ! !} \frac{x^{2 k}}{(2 k) !}
$$

with a suitably-defined $d_{n}$.

We show that the derivatives of the functions $Q_{n}(x, A)$ and $g_{n}(x, A)$ in (62) and 63) with respect to $x$ are non-negative.
First, the derivative of $e^{-x^{2} / 2} \tilde{I}_{n}(z x)$ with respect to $x$ in Taylor series form is seen to be

$$
\begin{aligned}
& \frac{d}{d x}\left(e^{-x^{2} / 2} \tilde{I}_{n}(z x)\right) \\
& =d_{n} e^{-x^{2} / 2} \sum_{k=1}^{\infty} \frac{(2 k-3) ! !}{(n+2 k-2) ! !} \frac{z^{2 k} x^{2 k-1}}{(2 k-2) !} \\
& \quad-d_{n} e^{-x^{2} / 2} \sum_{k=0}^{\infty} \frac{(2 k-1) ! !}{(n+2 k-2) ! !} \frac{z^{2 k} x^{2 k+1}}{(2 k) !} \\
& =d_{n} e^{-x^{2} / 2} \sum_{k=0}^{\infty}\left(\frac{z^{2 k+2}}{n+2 k}-z^{2 k}\right) \frac{(2 k-1) ! !}{(n+2 k-2) ! !} \frac{x^{2 k+1}}{(2 k) !}
\end{aligned}
$$

Using (91) in the definition of $Q_{n}(x, A)$, we see that

$$
\begin{aligned}
& \frac{d Q_{n}(x, A)}{d x} \\
& =\sum_{k=0}^{\infty} t_{n, k}(x) \int_{A}^{\infty}\left(\frac{z^{2 k+2}}{n+2 k}-z^{2 k}\right) z^{n-1} e^{-z^{2} / 2} d z
\end{aligned}
$$

where $t_{n, k}(x) \triangleq d_{n} e^{-x^{2} / 2} \frac{(2 k-1) ! !}{(n+2 k-2) ! !} \frac{x^{2 k+1}}{(2 k) !}$. We now show that the integral in 91) is non-negative, which implies that the derivative of $Q_{n}(x, A)$ with respect to $x$ is non-negative. We have

$$
\begin{aligned}
& \int_{A}^{\infty}\left(z^{2 k}\right) z^{n-1} e^{-z^{2} / 2} d z=\int_{A}^{\infty} e^{-z^{2} / 2}\left(\frac{z^{n+2 k}}{n+2 k}\right)^{\prime} d z \\
& =\frac{-A^{n+2 k} e^{-A^{2} / 2}}{n+2 k}+\int_{A}^{\infty}\left(\frac{z^{2 k+2}}{n+2 k}\right) z^{n-1} e^{-z^{2} / 2} d z
\end{aligned}
$$

where we used integration by parts in the last step. Rearranging the above equation, since $\frac{A^{n+2 k} e^{-A^{2} / 2}}{n+2 k}>0$, we see that the integral in (92) is non-negative.

For $g_{n}(x, A)$, a similar approach is used. Using (91) in the definition of $g_{n}(x, A)$, we see that

$$
\begin{aligned}
& \frac{d g_{n}(x, A)}{d x}= \\
& \sum_{k=0}^{\infty} t_{n, k}(x) \int_{A}^{\infty}\left(\frac{z^{2 k+2}}{n+2 k}-z^{2 k}\right) \frac{(z-A)^{2}}{2} z^{n-1} e^{-z^{2} / 2} d z
\end{aligned}
$$

A proof for the non-negativity of the integral in (94) is

$$
\begin{aligned}
\int_{A}^{\infty}\left(z^{2 k}\right) & \frac{(z-A)^{2}}{2} z^{n-1} e^{-z^{2} / 2} d z \\
= & \int_{A}^{\infty} \frac{(z-A)^{2}}{2} e^{-z^{2} / 2}\left(\frac{z^{n+2 k}}{n+2 k}\right)^{\prime} d z \\
= & \int_{A}^{\infty}\left(\frac{z^{2 k+2}}{n+2 k}\right) \frac{(z-A)^{2}}{2} z^{n-1} e^{-z^{2} / 2} d z \\
& -\int_{A}^{\infty}\left(\frac{z^{n+2 k}}{n+2 k}\right)(z-A) e^{-z^{2} / 2} d z
\end{aligned}
$$

where we used integration by parts in the last step. Rearranging the above equation, since $\left(\frac{z^{n+2 k}}{n+2 k}\right)(z-A) e^{-z^{2} / 2}>0$ for $z>A$, we see that the integral in 94 is non-negative. 


\section{APPENDIX C}

Since $X$ is uniform in $A_{N}$ and $\left|A_{N}\right|=N^{2}$, we have

$$
\begin{aligned}
P_{N} & =E\left[\|X\|^{2}\right]=\frac{1}{N^{2}} \sum_{n=1}^{N-1} \sum_{l=0}^{2 n}(n+0.5)^{2} \Delta^{2} \\
& =\frac{\Delta^{2}}{N^{2}} \sum_{n=1}^{N-1}(2 n+1)\left(n^{2}+n+\frac{1}{4}\right) \\
& =\frac{\Delta^{2}}{2}\left(N^{2}-\frac{1}{2}\left(1+\frac{1}{N^{2}}\right)\right) .
\end{aligned}
$$

To evaluate $\rho_{N}=E\left[X^{*} U\right] / P_{N}$, we consider

$$
E\left[X^{*}(X+U)\right]=E\left[E\left[X^{*}(X+U) \mid X\right]\right]
$$

Now $X^{*}(X+U) \mid X=0$ is zero with probability 1 , and $(X+U) \mid X=(n+0.5) \Delta e^{j(l+0.5) \theta_{n}}$ is uniform in the region specified in (42). So $X^{*}(X+U) \mid X=(n+0.5) \Delta e^{j(l+0.5) \theta_{n}}$ is uniform in the region

$$
\begin{aligned}
\{(r \cos \theta, r \sin \theta): n(n+0.5) & \leq \frac{r}{\Delta^{2}} \leq(n+1)(n+0.5) \\
-0.5 & \left.\leq \frac{\theta}{\theta_{n}} \leq 0.5\right\}
\end{aligned}
$$

A calculation shows that

$$
\begin{aligned}
E\left[X^{*}(X+U) \mid X\right. & \left.=(n+0.5) \Delta e^{j(l+0.5) \theta_{n}}\right] \\
= & \Delta^{2}\left(n^{2}+n+\frac{1}{3}\right) \operatorname{sinc} \frac{\pi}{2 n+1}
\end{aligned}
$$

where $\operatorname{sinc} x=\frac{\sin x}{x}$. Therefore, we have

$$
\begin{aligned}
E & {\left[X^{*}(X+U)\right] } \\
& =\frac{1}{N^{2}} \sum_{n=1}^{N-1} \sum_{l=0}^{2 n} \Delta^{2}\left(n^{2}+n+\frac{1}{3}\right) \operatorname{sinc} \frac{\pi}{2 n+1} \\
& =\frac{\Delta^{2}}{N^{2}} \sum_{n=1}^{N-1}(2 n+1)\left(n^{2}+n+\frac{1}{3}\right) \operatorname{sinc} \frac{\pi}{2 n+1} .
\end{aligned}
$$

Since $E\left[X^{*}(X+U)\right]=E\left[\|X\|^{2}\right]+E\left[X^{*} U\right]$, we have

$$
\begin{aligned}
\rho_{N} P_{N}=\frac{\Delta^{2}}{N^{2}} \sum_{n=1}^{N-1}(2 n+1) & {\left[\left(n^{2}+n+\frac{1}{3}\right) \operatorname{sinc} \frac{\pi}{2 n+1}\right.} \\
& \left.-\left(n^{2}+n+\frac{1}{4}\right)\right] .
\end{aligned}
$$

The sequence $a_{n}=\left(n^{2}+n+1 / 4\right)-\left(n^{2}+n+\right.$ $1 / 3) \operatorname{sinc}(\pi /(2 n+1))$ is increasing and converges to $\frac{\pi^{2}-2}{24} \leq$ 0.33 with $a_{1} \geq 0.32$. We thus have

$$
-0.33\left(1-\frac{1}{N^{2}}\right) \leq \frac{\rho_{N} P_{N}}{\Delta^{2}} \leq-0.32\left(1-\frac{1}{N^{2}}\right) .
$$

From (97), using $P_{N}=\frac{\Delta^{2} N^{2}}{2}\left(1-O\left(1 / N^{2}\right)\right)$, we have

$$
-0.66\left(1-O\left(1 / N^{2}\right)\right) \leq \rho_{N} N^{2} \leq-0.64\left(1-O\left(1 / N^{2}\right)\right) .
$$

The expression inside the second $\log$ term in (48) is

$$
\frac{N^{2}}{\alpha}-\frac{P_{N}^{2}\left(1+\rho_{N}\right)^{2}}{N^{2} \Delta^{2}} \text {. }
$$

Since $P_{N}=\frac{\Delta^{2} N^{2}}{2}\left(1-0.5 / N^{2}-O\left(1 / N^{4}\right)\right)$, we have

$$
\frac{P_{N}^{2}}{N^{2} \Delta^{2}}=P_{N} \frac{P_{N}}{\Delta^{2} N^{2}}=\left(\frac{N^{2}}{\alpha}-1\right)\left(1-\frac{0.5}{N^{2}}-O\left(1 / N^{4}\right)\right)
$$

where we have also used $N^{2}=\alpha\left(1+P_{N} / 2\right)$. We arrive at

$$
\begin{aligned}
\frac{N^{2}}{\alpha} & -\frac{P_{N}^{2}\left(1+\rho_{N}\right)^{2}}{N^{2} \Delta^{2}} \\
& =\frac{N^{2}}{\alpha}-\left(\frac{N^{2}}{\alpha}-1\right)\left(1-0.5 / N^{2}-O\left(1 / N^{4}\right)\right)\left(1+\rho_{N}\right)^{2} \\
& \geq 1+\frac{1.82}{\alpha}+O\left(1 / N^{2}\right)
\end{aligned}
$$

using $\rho_{N} N^{2} \geq-0.66\left(1-O\left(1 / N^{2}\right)\right)$. This explains the final analytical lower bound given in (49). 